CMS endcap RPC gas gap production for upgrade

This content has been downloaded from IOPscience. Please scroll down to see the full text. 2012 JINST 7 P11013

(http://iopscience.iop.org/1748-0221/7/11/P11013)

View the table of contents for this issue, or go to the journal homepage for more

Download details:

IP Address: 157.193.59.60

This content was downloaded on 25/08/2014 at 12:22

Please note that terms and conditions apply. 


\title{
CMS endcap RPC gas gap production for upgrade
}

\author{
S.K. Park, ${ }^{a, 1}$ S. Choi, ${ }^{a}$ B. Hong, ${ }^{a}$ Y. Gun Jeng, ${ }^{a}$ M. Kang, ${ }^{a}$ K.S. Lee, ${ }^{a}$ K.-S.Sim, ${ }^{a}$ \\ A. Colaleo, ${ }^{b}$ G. Pugliese,${ }^{b}$ F. Loddo, ${ }^{b}$ C. Calabria, ${ }^{b}$ M. Maggi, ${ }^{b}$ P. Verwillingen, ${ }^{c}$ \\ U. Berzano, ${ }^{c}$ C. Carrillo, ${ }^{e}$ A. Aleksandrov,${ }^{f}$ V. Genchev, ${ }^{f}$ P. laydjiev,${ }^{f}$ M. Rodozov, ${ }^{f}$ \\ M. Shopova ${ }^{f}$ and G. Sultanov ${ }^{f}$

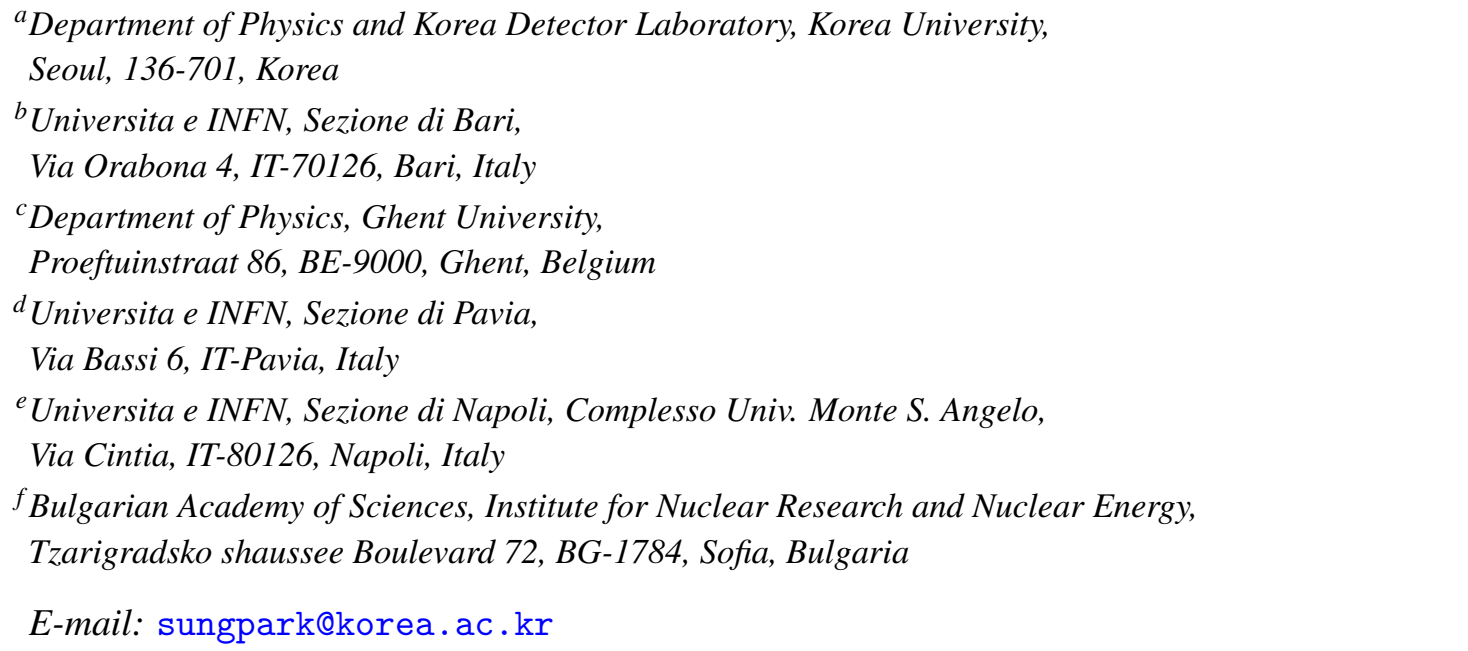

ABSTRACT: The CMS experiment will install a RE4 layer of 144 new Resistive Plate Chambers (RPCs) on the existing york YE3 at both endcap regions to trigger high momentum muons from the proton-proton interaction. In this paper, we present the detailed procedures used in the production of new RPC gas gaps adopted in the CMS upgrade. Quality assurance is enforced as ways to maintain the same quality of RPC gas gaps as the existing 432 endcap RPC chambers that have been operational since the beginning of the LHC operation.

KEYWORDS: Detector design and construction technologies and materials; Resistive-plate chambers; Trigger detectors

\footnotetext{
${ }^{1}$ Corresponding author.
} 


\section{Contents}

1 Introduction 1

2 Production procedure $\quad 3$

2.1 Mechanical components 3

2.2 Polymerization 4

2.3 High voltage test 5

3 Packing and transportation 5

4 Conclusions 6

\section{Introduction}

Resistive Plate Chamber (RPC) is a dedicated fast muon trigger for the CMS experiment at LHC [1]. Installed both in the barrel and in the endcap region it was originally planned to cover a rapidity up to $\eta=2.1$. However in the endcap region, the original four layers were reduced to three which have been installed on the faces of the iron return yokes. It consequently reduces the rapidity coverage up to $\eta=1.6$.

Completion of the endcap RPC system with four layers will be achieved by addition of one more RPC layer to the existing layers. The new 144 RPCs will form this additional layer called RE4. And it will be installed on the face of the existing yoke YE3. And it will restore the trigger coverage up to $\eta=2.1$ as planned.

For the RPCs to provide an efficient muon trigger to the CMS experiment, the gas gaps must meet the CMS requirements for RPCs as shown in table 1. To meet these performance requirements, we build the gaps with two most important considerations in mind: an overall mechanical stability and an uniform electric field over the active gas volume.

In the CMS RPCs, one side of the bakelite plate is coated with graphite to make the resistive electrode. Two resistive electrodes are separated by $2 \mathrm{~mm}$ spacers to form the parallel plate gas

Table 1. CMS RPC requirements.

\begin{tabular}{|ll|}
\hline Parameters & Allowable ranges \\
\hline Efficiency & $>95 \%$ \\
Time resolution & $\leqslant 1 \mathrm{~ns}$ \\
Average cluster size & $\leqslant 2 \mathrm{strips}$ \\
Rate capability & $2 \mathrm{kHz} / \mathrm{cm}^{2}$ \\
Mean avalanche charge & $2.5-5 \mathrm{pC}$ \\
\hline
\end{tabular}




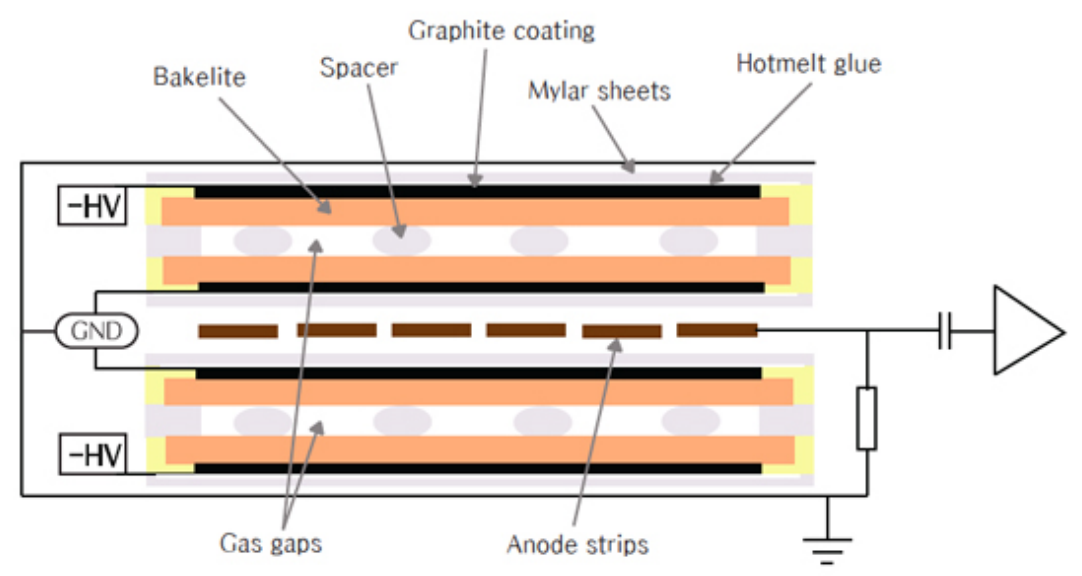

Figure 1. Cross sectional view of a CMS double gap RPC.
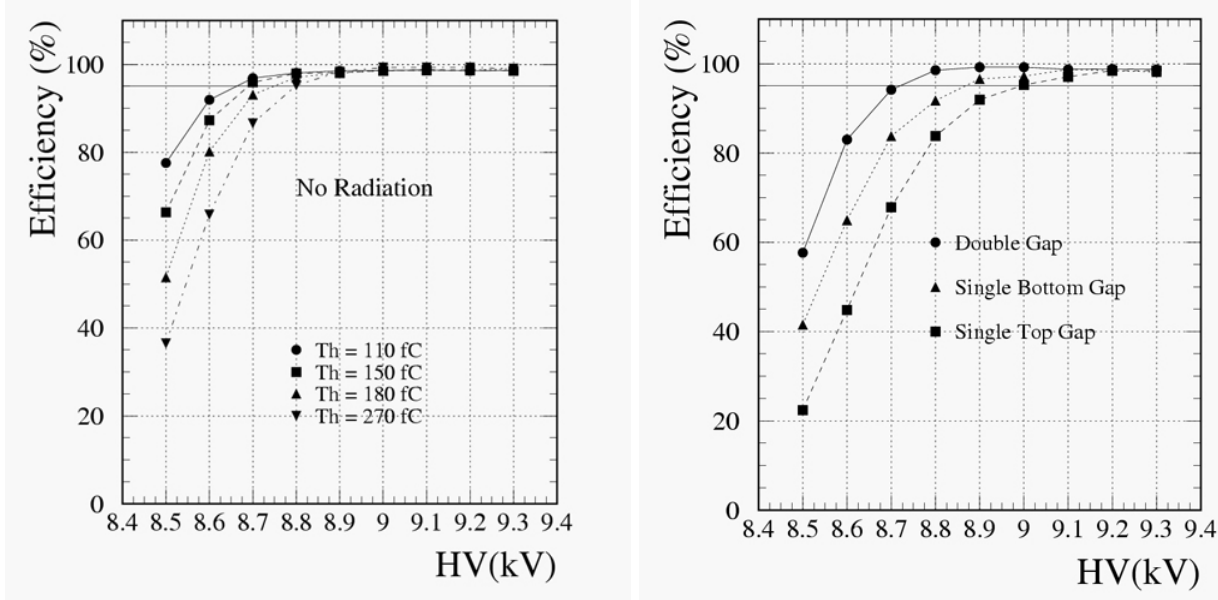

Figure 2. The RPC efficiencies in terms of charge thresholds (left), and number of gaps (right).

gaps. For higher trigger efficiency the RPC gaps are in double layers. The basic structure of the CMS double gap RPC is shown in figure 1.

In this paper, we describe each step according to the technology that Korea Detector Laboratory (KODEL) developed for the endcap RPCs which have been already installed and operational since the beginning of the LHC operation. The high quality of the CMS RPC gaps are well captured in figure 2 as in [2]. 


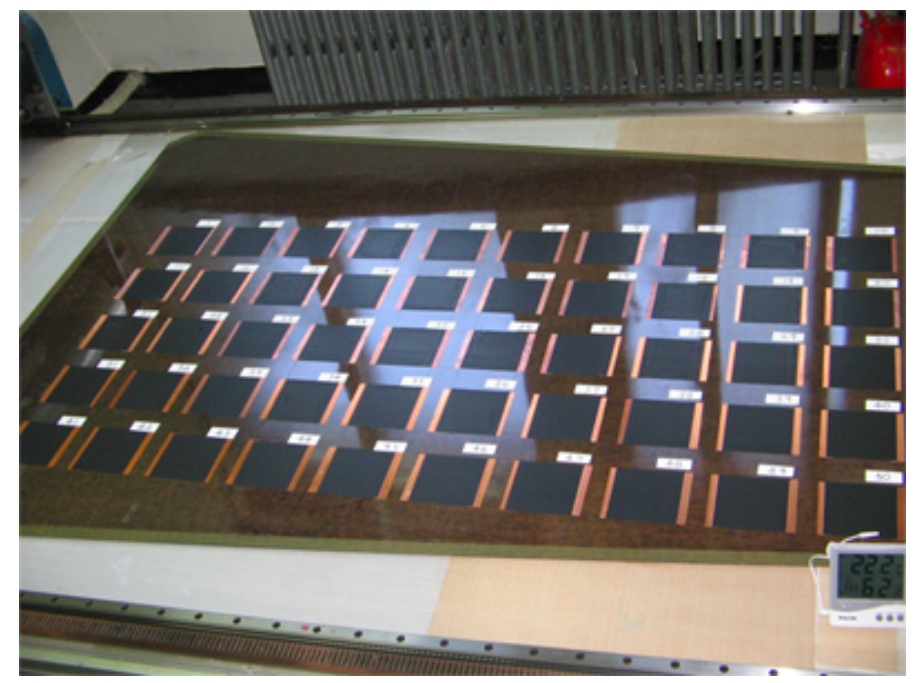

Figure 3. Arrangement of 50 samples of bakelite pieces to check the surface resistivity.

\section{Production procedure}

\subsection{Mechanical components}

The electrode production is the first step in the production of the gas gaps. A thin layer of graphite is coated on the bakelite sheet by a silkscreen process. The thickness of the graphite layer is well maintained by the $20 \mu \mathrm{m}$ thick silk screen mesh. The surface resistivity of the graphite layer ranges from 100 to $250 \mathrm{k} \Omega$ /square. To ensure the values and homogeneity across the electrode 50 pieces of square bakelite were prepared and the surface resistivity was measured from each sample as shown in figure 3.

The gas gap assembly is the next step once the electrodes are prepared. The gas gaps are assembled with two electrodes and spacers. The coin shaped spacers are placed between two electrodes to form a uniform separation of $2 \mathrm{~mm}$ between the electrodes, and the four edges of the gas gap are sealed with four edge strips. The mechanical support of the gas gap is provided by the spacers placed $10 \mathrm{~cm}$ apart from each other in two orthogonal directions. The edge strips that seal the edges of the gas gap make the gas volume leak-tight. The spacers and edge strips are placed in pre-determined locations guided by a special jig and bonded to the electrodes using epoxy with a curing time of 24 hours. To achieve the maximum bonding strength between the spacers and electrodes, the gaps are placed in a device where an additional $30 \mathrm{hPa}$ pressure to ATM is applied.

The mechanical stability check of the gas gap follows after 24 hours of hardening to see the stability of the bonding and the gas tightness of the gas volume. During this test the gas volume is over-pressured with $20 \mathrm{hPa}$. For a gas gap to be qualified the loss of the measured pressure of the gap should be less than $0.2 \mathrm{hPa}$ during a 10-minute of test period. Additional requirement for gas gaps to pass the test is that no spacers should lose its bonding strength between two electrodes when the $20 \mathrm{hPa}$ over-pressure is applied to the gas volume. All these mechanical tests are carried out by a robot built at KODEL and the test results as shown in figure 5 are stored in a CMS central ORACLE data base. 


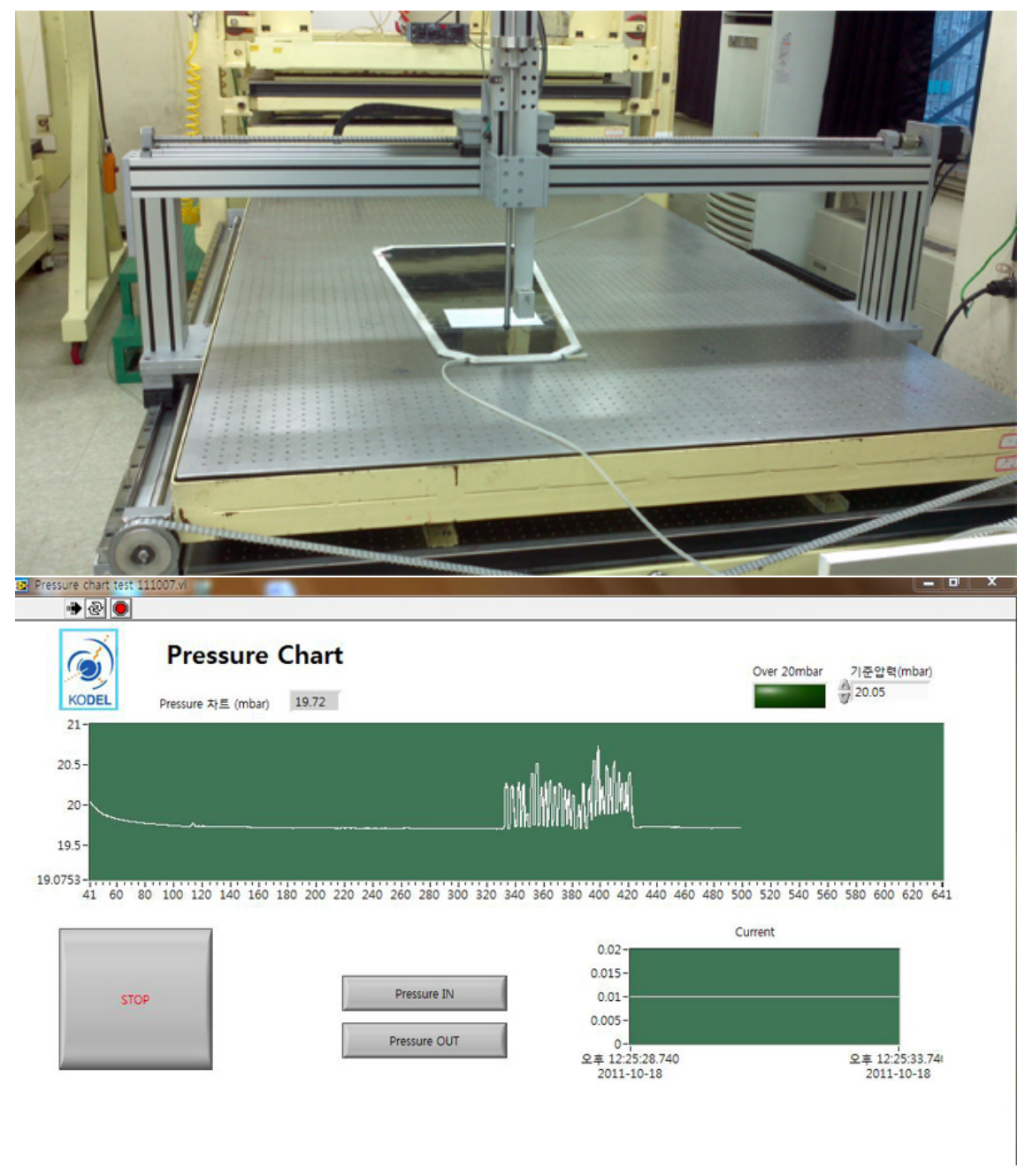

Figure 4. KODEL robot performing the pressure test. The dimensions of the table are $175 \mathrm{~cm} \times 250 \mathrm{~cm}$ (top). The result of the pressure test is shown (bottom).

\subsection{Polymerization}

The linseed oil treatment will follow once the gas gap passes the mechanical stability test. During this process the entire gas volume is filled with linseed oil. Once dried with air the oiled surface forms the polymerized layer. It turns out that this polymerized layer on the surface of the bakelite inside the gas volume reduces the spurious noise by a factor of 10 in the avalanche operation mode [3]. This procedure needs a cautious preparation due to the pressure built up by the linseed oil filling the gas gap. The pressure within the gap should remain less than $20 \mathrm{hPa}$ over ATM to ensure that the gas gap is not over-pressured to the limit of its epoxy bonding strength.

The RE4 has six different shapes and sizes. Accommodating the variety of physical dimensions of the gas gaps to the oil coating facility is a difficult task due to the different orientations of the gas inlets and outlets. Also the drying period of the linseed oil surface in the gas volume becomes a significant constraint in production schedule. 


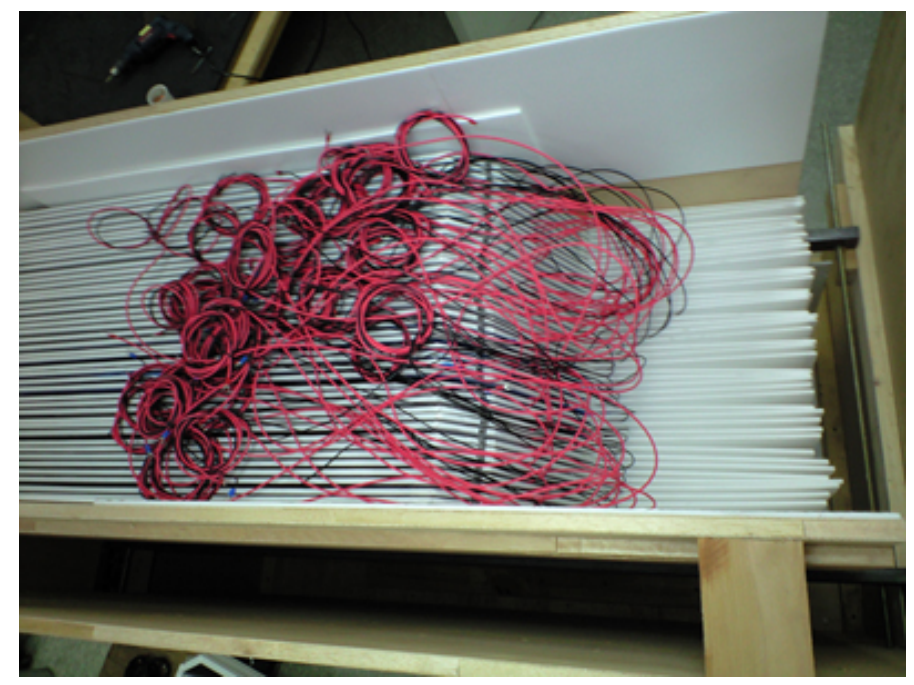

Figure 5. A wooden box is designed to provide extra protections.

\subsection{High voltage test}

The high voltage test is the next step once the gas volume treated with linseed oil is dried. The current drawn by the gas gap with high voltage applied is a good indicator of any electrical problems inside the gas volume.

The gas mixture for the current measurement under high voltage is $95 \%$ tetrafluoroethane, $5 \%$ isobutane. The gas volume is purged at a rate of $20 \mathrm{l} / \mathrm{h}$ before applying high voltage. It amounts to 15 times the volume of the gas gap. The total duration of the current measurement is about 7 days including the purging time.

At the beginning of the test, the high voltage is set to $1 \mathrm{kV}$ to check for any electrical misconnection. If the gaps hold at $1 \mathrm{kV}$, then every 30 minutes, the $\mathrm{HV}$ is increased by $1 \mathrm{kV}$ up to 6 $\mathrm{kV}$. At each HV setting, the currents are measured and recorded in a data sheet. From $6 \mathrm{kV}$ the HV is raised up to the maximum $10 \mathrm{kV}$. From $8.4 \mathrm{kV}$, the increasing step is $200 \mathrm{~V}$, finer than the $1 \mathrm{kV}$ step to see the current behaviors in the operation voltages. Once the HV has reached the maximum $10 \mathrm{kV}$, it is lowered to $9.6 \mathrm{kV}$ for a test period of 96 hours.

\section{Packing and transportation}

Every 60 qualified gas gaps are vertically mounted into a wooden box equipped with four preloaded bars which were designed to press the surface of the gas gaps horizontally. Polystyrene foam sheets of $5 \mathrm{~mm}$ thickness are placed between the layers of the gas gaps to absorb shocks which could occur during transportation. Extra protection from unexpected moisture is provided by wrapping the whole 60 gas gaps with a thin vinyl film. The pipes for the gas inlets and outlets are left open to adapt to sudden variations of pressure during air transport. 


\section{Conclusions}

We report that 144 new RPCs will be produced according to the same procedure that Korea Detector Laboratory (KODEL) developed for the CMS endcap RPC stations of RE1, RE2 and RE3. We conclude that the production and subsequent quality assurance described here has been proven to be effective for the mass production of the large RPC detectors for the CMS experiment.

\section{Acknowledgments}

We acknowledge the support of Korea University and the Korean Research Foundation.

\section{References}

[1] CMS collaboration, The Muon Project, Technical Design Report, CERN-LHCC 97-32, December 1997.

[2] S. Ahn et al., Threshold dependence of strip clusters for the forward region resistive plate chamber of the CMS/LHC experiment, Nucl. Instrum. Meth. A 508 (2003) 147.

[3] S. Park et al., Beam test results of CMS RPCs at high eta region under high-radiation environment, Nucl. Instrum. Meth. A 533 (2004) 37. 